\title{
Real-time Drive Cycle Simulation of Automotive Climate Control Sys- tem
}

\author{
Anand Pitchaikani, Kingsly Jebakumar S, Shankar Venkataraman, S. A. Sundaresan \\ Emmeskay, Inc \\ 47119 Five Mile Road, Plymouth, MI 48170, USA \\ anandp@emmeskay.com_kingsly@emmeskay.com sasundaresan@emmeskay.com
}

\begin{abstract}
Technologies like hardware-in-the-loop simulation (HILS) can play a significant role in reducing the product development life cycle. An essential requirement for HILS is the availability of system models that are capable of running in real-time. This paper demonstrates a closed loop real-time simulation of a vehicle with a climate control system model developed in Modelica. This paper details the modeling aspects of the vehicle system and climate control system using the object-oriented, acausal modeling language Modelica. To demonstrate the real-time simulation capabilities of the developed models, they were integrated with a controller model developed in the Simulink environment and the integrated model was then simulated in an Opal-RT real-time environment. This paper describes the models developed and the tool-chain used to achieve real-time simulation. The real-time simulation results as well as the performance results are also presented. This paper demonstrates Modelica's capabilities in creating models for real-time vehicle climate control system simulations.
\end{abstract}

Keywords: climate control system; vapor cycle; closed-loop simulation; real-time simulation;

\section{Introduction}

The development of system simulation models for vehicle as well as the air-conditioning system is extensively described in various previous works $[1,2$ and 4]. The challenge lies in developing these models so as to balance the need for real time capability and reasonable accuracy. The real-time closed-loop simulation of the plant model along with the controller model is a major validation milestone in the controller development process.

A conventional internal combustion engine powered vehicle is considered in this work. The system includes an engine driven compressor and a va- por compression refrigeration cycle based Heating, Ventilation and Air-Conditioning (HVAC) system. Models for vehicle subsystems and climate control system subsystems are required to study the impact of the vehicle climate control system over a simulated drive cycle. The vehicle subsystems (engine, transmission, drive train, chassis and accessory drive) and the HVAC components (compressor, condenser, evaporator and expansion valve) need to be modeled. The literature reports the development of models for HVAC components $[1,2]$. The accuracy levels of these reported models differ as they address different needs like design of climate control system or simulation of overall vehicle with climate control system. Though the real-time simulation of Modelica ${ }^{\circledR}$ models was demonstrated successfully for vehicle system models [4], it has not been reported for a vehicle model with climate control system. The real time simulation of vehicle model with climate control system components will help the developers of the climate system controllers by enabling Hardware-in-the-Loop simulation of varying complexities. Different controller hardware can be tested with these real time models on an HILS platform.

The controller model was available as Simulink ${ }^{\circledR}$ S-function along with the object code. The assembled vehicle and climate control system models in Modelica were converted to a Simulink C-file Sfunction using Dymola ${ }^{\circledR}$ [9]. The plant and controller S-function models were integrated in the Simulink environment. This closed loop model consisting of the two S-functions was downloaded to OpalRT's [11] target processor. The controller model was simulated in the target itself due to non-availability of the controller hardware. This real time simulation verified the real time capability of the developed plant models as well as ability of the controller to achieve the control targets. This takes the overall controller development process one step closer to closed loop HIL simulation.

Though literature is available for such real time simulations using Modelica models [5] in other do- 
mains, there is very little reported in the area of HVAC system. A HVAC system is a multi-domain system straddling mechanics, fluid mechanics and thermal engineering.

In this paper the details involved in modeling the vehicle as well as the climate control system components are described. The simulation was carried out by parameterizing the vehicle and climate control system to represent a conventional mid sized sedan.

\section{Tool-chain}

The plant models were developed using the Modelica language in the Dymola 6.1 environment. The development re-used most of the components from the Modelica Standard Library (MSL). The special needs were satisfied by creating additional custom models in Modelica. The Dymola-Simulink interface is used to create the S-function of the developed plant models. The controller S-function and plant model S-function are integrated in MATLAB ${ }^{\circledR}$ $\mathrm{R} 2006 \mathrm{~b}$ version. The integrated model is converted to a real-time model by using RT-LAB ${ }^{\mathrm{TM}}$ 8.1.7. This real-time model is downloaded into the TestDrive ${ }^{\mathrm{TM}}$ system that operates on QNX ${ }^{\circledR}$ 6.3. The real-time simulation is carried out in the above mentioned TestDrive system. The above tools were used based on their applicability to the automotive domain.

\section{Plant Models}

The goal of this work was to model a conventional engine driven vehicle with engine driven HVAC system. The vehicle subsystems (driver, controller, engine, transmission and chassis) and the vehicle airconditioning subsystems (compressor drive, HVAC systems, cabin and atmosphere) are modeled in this work. Most of these models are developed using simple formulations keeping the real-time requirements in mind. The overall plant model architecture (Figure 1) in Modelica is made such that all the main subsystems are replaceable. This architecture provides flexibility to choose different implementations for each subsystem. In this way, the same model can be configured to model different vehicle systems easily. However, only the real-time capable configuration is described in this paper.

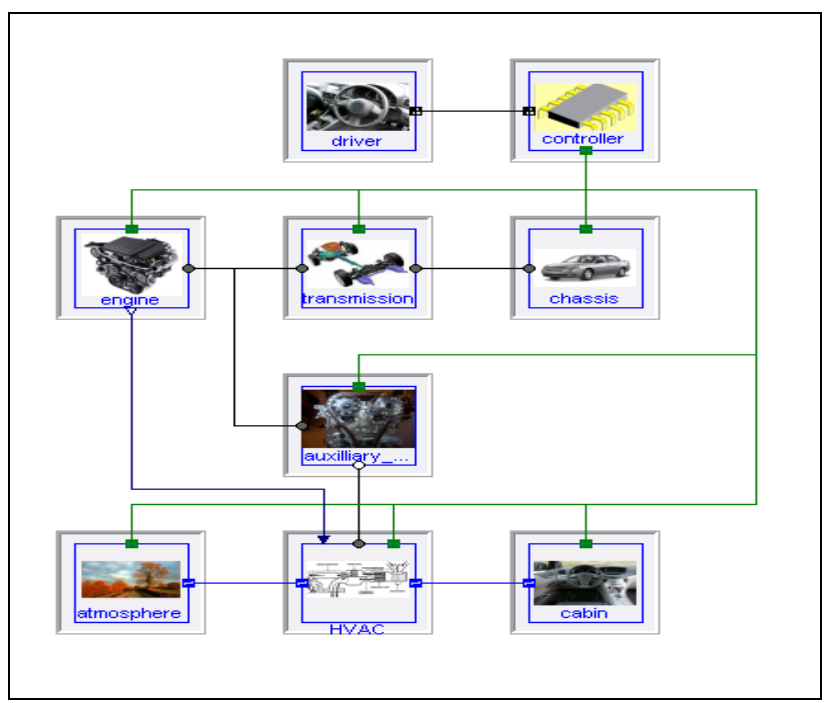

Figure 1 System Architecture

\subsection{Vehicle System Models}

\subsubsection{Driver}

The driver model generates the input signals like throttle, brake and clutch commands. The driver model takes advantage of the acausal nature of the Modelica language for setting up the inverse problem: taking the drive cycle vehicle speed command as input and then computing the clutch, accelerator pedal and brake pedal commands required to follow the desired drive cycle input. In other words, the model is "backward driven" (at least with respect to the longitudinal vehicle dynamics). The model is built with the capability of idling the engine when the vehicle is at rest. These driver command signals are sent to the controller through the driver bus.

\subsubsection{Controller}

The controller model processes the driver commands and plant sensor outputs and generates control signals for the engine, transmission and the HVAC system. As a simple engine model is used in this study, the engine control logic is a simple pass through in the controller model. The climate system controller is a part of the controller model. The control signals are sent to the plant through the system bus. The system bus is an expandable connector from which all other components can access the required control signals. In the case of a "forward-driven" model, the controller model would quite be complex and play an important role in driver inputs like throttle, brake, clutch and gear command. Because of the "backward driven" driveline model, most of the complexity in the controller model is related to control of the HVAC components. 


\subsubsection{Engine}

The engine model delivers torque based on commanded throttle from the driver. This model incorporates performance maps in the form of look-up tables. The torque-speed map which provides a minimum and maximum limit of the torque for every speed is used where minimum corresponds to 0 throttle and maximum corresponds to 1 throttle. The fuel consumption map is based on the torque and speed. This formulation will be very useful for fuel economy studies. Also such minimal data will be very easy to get for parameterizing any type of engine.

\subsubsection{Transmission}

The transmission model is a six-speed manual type encompassing clutch, synchronizers and final drive. The gear, clutch and inertia models of MSL are used to construct the transmission model. The current gear selection is given by a gear-shift map based on throttle command and engine speed. A simple gear selector model is included in the transmission model. This model includes a shift map that consists of threshold speed limits for upshifts and downshifts, at the given throttle. Based on the throttle and engine speed, the gear selector will select a gear number and engage the appropriate synchronizer. The final drive is modeled as a simple gear. A simple clutch model is used to represent the clutch that engages and disengages the engine from the driveline. This model will capture the torque ratio introduced by the transmission and effective inertia of the transmission based on the gear engaged.

\subsubsection{Chassis}

The chassis model handles the longitudinal dynamics of the vehicle by modeling vehicle mass, aerodynamic drag and rolling resistance. The driver brake command is converted to brake torque and is sent to the wheels. Drag and rolling resistance are modeled using representative equations. The chassis model gets the torque generated in the engine model through the transmission and final drive. The vehicle speed from the chassis model is put on to the system bus.

\subsection{Climate Control System Models}

The climate control model includes the cabin, atmosphere, auxiliary drive and HVAC components as shown in Figure 2. The climate control components handle air flow as well as refrigerant flow. Expandable HVAC connectors are used in the subsystem level so that the separate interfaces used for air and refrigerant are not visible at the top-most level.

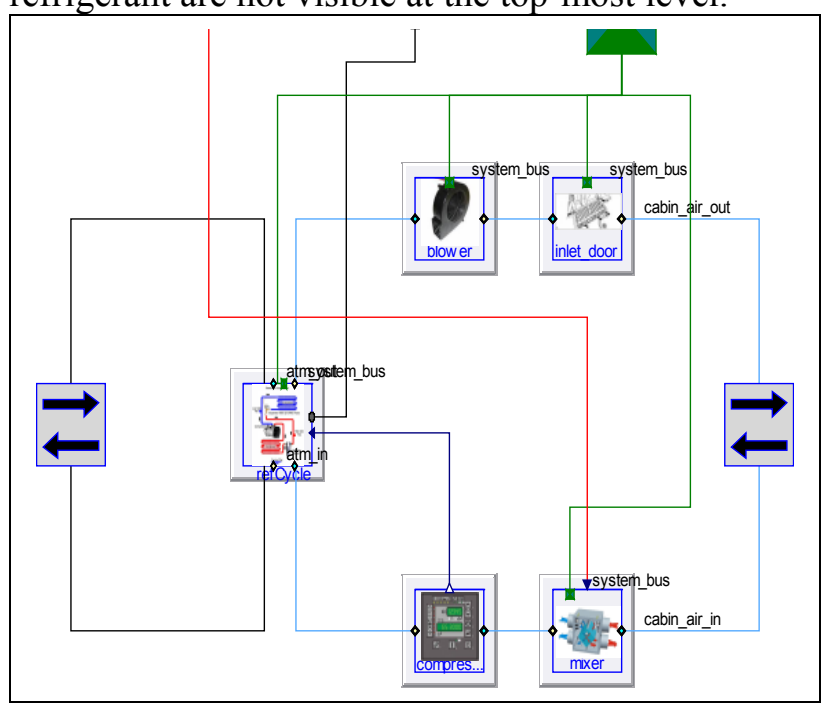

Figure 2 Climate Control System Models

\subsubsection{Cabin}

The single zone cabin is modeled as a closed thermal volume surrounded by a metal frame mass. Solar heating effects are included in the model. The amount of solar heating will vary with the ambient conditions chosen for the simulation. The heat capacity of the cabin metal mass is captured in the model. The heat transfer from metal frame to the interior is through convection. For typical closed cabin climate control systems, it was felt that relative humidity effects will not be significant and hence were not modeled. Air flow inside the cabin is modeled using custom made air flow connectors.

\subsubsection{Atmosphere}

The atmosphere is modeled as an infinite sourcesink model which fixes the vehicle ambient conditions like temperature, pressure, air density etc. This reference data is used in the HVAC models to compute the heat transfer and related variables associated with air flow over the condenser and evaporator. For the sake of simplicity, the mass flow rate of air that blows over the condenser is set as a constant in the atmosphere model itself. The mass flow rate of air that blows over the evaporator will be fixed by the blower model and the atmosphere or the cabin will supply that air flow based on the recirculation command.

\subsubsection{Auxiliary Drive}

The auxiliary drive model captures the gearing and clutch between the engine shaft and the HVAC compressor drive shaft. It represents the engine-driven compressor configuration in which a portion of 
the engine power is used to drive the HVAC compressor. The compressor off signal from the climate system controller will disengage the clutch modeled in the auxiliary drive. This model captures the load applied by the HVAC system on the engine which is crucial in estimating the fuel economy accurately.

\subsubsection{HVAC}

The HVAC components form the core of the climate control system. They include models for refrigeration cycle, refrigerant properties, blower, inlet door and heater as shown in Figure 2 and Figure 3. The refrigeration cycle models include the vaporcycle refrigeration components like evaporator, expansion valve, condenser and compressor. The refrigerant expansion, compression and heat transfer processes have been captured in the models. The refrigerant flow and air flow interact thermally by using thermal connectors of MSL.

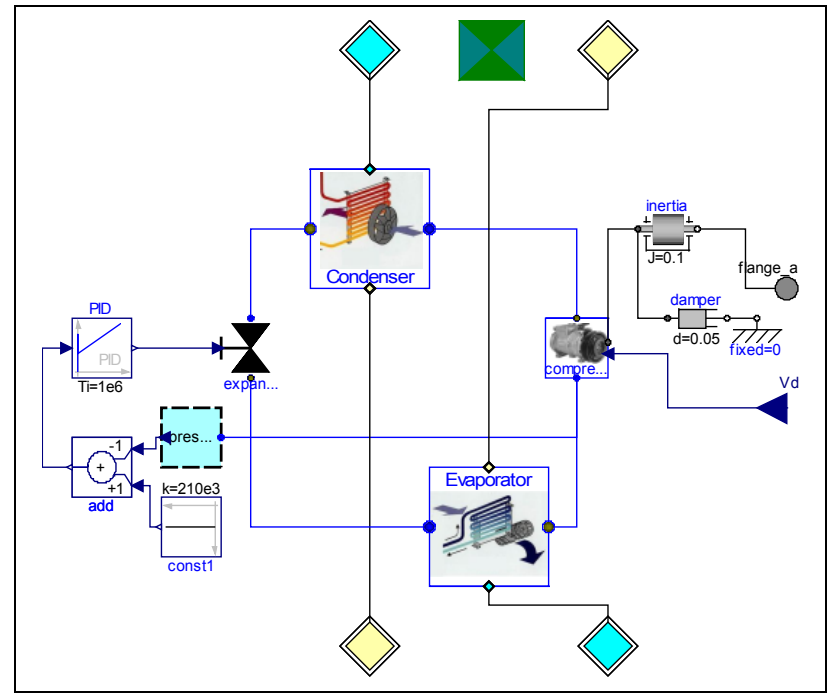

Figure 3 HVAC Components

\subsubsection{Compressor}

HVAC compressor is modeled using isentropic relationships as shown below. Compressor is characterized by its volumetric efficiency and isentropic efficiency. Provision has been given to vary its displacement volume as required.

For model robustness we ensured that the compressor model could handle the following special conditions:

- Suction pressure greater than discharge pressure

- Negative compressor speed

$$
\begin{gathered}
\dot{m}=\frac{\omega}{2 \pi} \eta_{v} V_{d} \rho_{\text {suction }} \\
M=\frac{\gamma \eta_{v} P_{\text {suction }} V_{d}}{2 \pi \eta_{\text {isen }} \eta_{\text {mech }}(1-\gamma)}\left(r^{\frac{\gamma-1}{\gamma}}-1\right) \text { Where }
\end{gathered}
$$

$\mathrm{m}$ - Refrigerant flow rate $(\mathrm{kg} / \mathrm{s})$

$\omega$ - Compressor rotational speed $(\mathrm{rad} / \mathrm{s})$

$\rho_{\text {suction }}-$ Refrigerant density at suction side $(\mathrm{kg} / \mathrm{m} 3)$

$\mathrm{M}$ - compressor torque (Nm)

$\mathrm{r}$ - Pressure ratio

$\mathrm{P}_{\text {suction }}-$ Suction pressure $(\mathrm{N} / \mathrm{m} 2)$

$\gamma$-Ratio of specific heat capacities of the refrigerant

$\mathrm{V}_{\mathrm{d}}$ - Displacement volume (m3)

$\eta_{\mathrm{v}}-$ Volumetric efficiency

$\eta_{\text {isen }}$-Isentropic efficiency

$\eta_{\text {mech }}-$ Mechanical efficiency

\subsubsection{Condenser and Evaporator}

The condenser and evaporator are modeled as lumped volume elements to emulate the heat exchange between the refrigerant medium and air (see Figure 4). This model incorporates the two important heat exchange mechanisms as given below.

- Convection between refrigerant and pipe wall

- Convection between pipe wall and air

The convective heat transfer coefficients on the refrigerant side are estimated based on the refrigerant quality and other thermal coefficients [7]. The pipe wall heat capacity is captured in the model. The convective heat transfer coefficient is modeled to have two values - one for low air mass flow rates and another for high air mass flow rates. The pressure loss inside the heat exchangers is assumed to be negligible and hence the entire heat exchanger volume is lumped into a single volume. We accepted these assumptions as reasonable in order to achieve real-time performance.

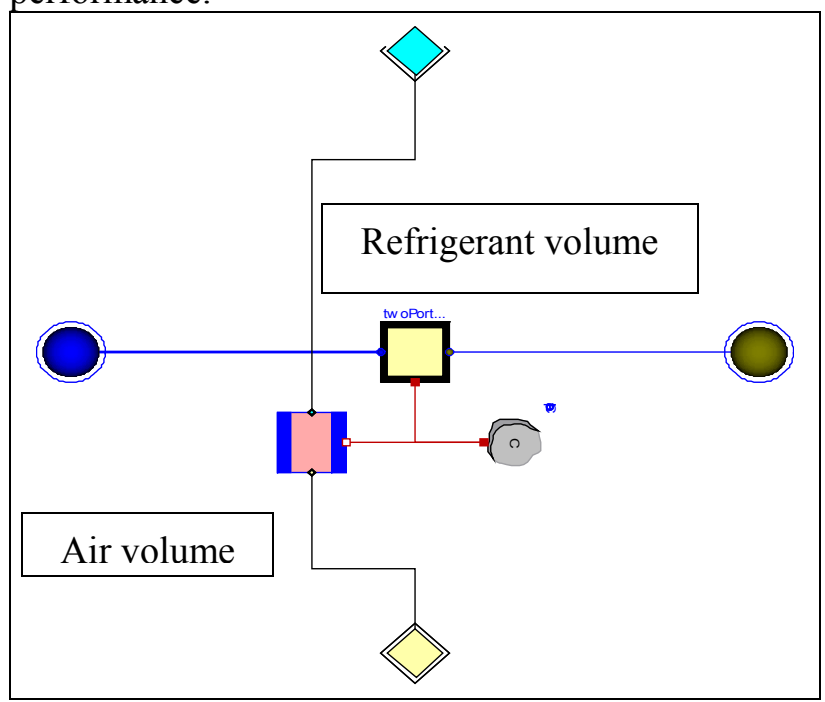

Figure 4 Condenser and Evaporator

The processes modeled in the condenser and evaporator volume models can be summarized as below. 
$\frac{d m_{v o l}}{d t}=\dot{m}_{i}-\dot{m}_{o}$

$\frac{d\left(m_{v o l}\left(h_{v o l}-P_{v o l} v_{v o l}\right)\right)}{d t}=\dot{m}_{i} h_{i}-\dot{m}_{o} h_{o}+U_{i} A\left(T_{w}-T_{v o l}\right)$

$C \frac{d T_{w}}{d t}=U_{o} A\left(T_{a m b}-T_{w}\right)-U_{i} A\left(T_{w}-T\right)$ Where

$\mathrm{m}_{\mathrm{vol}}$ - Mass of refrigerant in the volume $(\mathrm{kg})$

$\mathrm{m}_{\mathrm{i}}$ - Refrigerant inlet mass flow rate $(\mathrm{kg} / \mathrm{s})$

$\mathrm{m}_{\mathrm{o}}$ - Refrigerant outlet mass flow rate $(\mathrm{kg} / \mathrm{s})$

$\mathrm{h}_{\mathrm{vol}}$ - Specific enthalpy of refrigerant in the volume $(J / k g)$

$\mathrm{P}_{\mathrm{vol}}-$ Pressure of refrigerant in the volume $\left(\mathrm{N} . \mathrm{m}^{2}\right)$

$v_{\mathrm{vol}}$ - Specific volume of refrigerant in the volume $(\mathrm{m} 3 / \mathrm{kg})$

$\mathrm{h}_{\mathrm{i}}-$ Specific enthalpy of incoming refrigerant flow $(\mathrm{J} / \mathrm{kg})$

$\mathrm{h}_{\mathrm{o}}$ - Specific enthalpy of outgoing refrigerant flow $(\mathrm{J} / \mathrm{kg})$

$\mathrm{U}_{\mathrm{i}}-$ Refrigerant side convection heat transfer coefficient $(\mathrm{W} / \mathrm{m} 2 \mathrm{~K})$

$\mathrm{U}_{\mathrm{o}}-$ Air side convection heat transfer coefficient (W/ $m 2 K)$

$\mathrm{T}_{\mathrm{vol}}$-Refrigerant temperature in the volume $(K)$

$\mathrm{T}_{\mathrm{w}}$ - Pipe wall temperature $(K)$

$\mathrm{T}_{\text {amb }}-$ Ambient air temperature $(K)$

$\mathrm{C}$ - Specific heat capacity of pipe $(\mathrm{J} / \mathrm{K})$

\subsubsection{Expansion Valve}

The expansion valve is modeled based on empirical relationship for flow as a function of pressure drop [3]. It can handle the choked mass flow based on sub-cooled entry conditions. The expansion valve is modeled as a variable area flow device. The valve area is an input to the model. A PID controller is used to control the flow area to maintain near constant suction pressure. This will ensure a proper functioning of the compressor model.

\subsubsection{Refrigerant Properties}

The refrigerant type considered in this work is $\mathrm{R} 134 \mathrm{a}$. The refrigerant properties are generated using property relationship curves available in the literature [6] and used in the evaporator and condenser models. The refrigerant properties were represented by data tables created from the complex empirical relationships (see Figure 5). The tables specify the enthalpy, pressure and quality (mass fraction) for the given density and temperature. The resulting model is computationally less intensive and hence capable for real-time simulation.

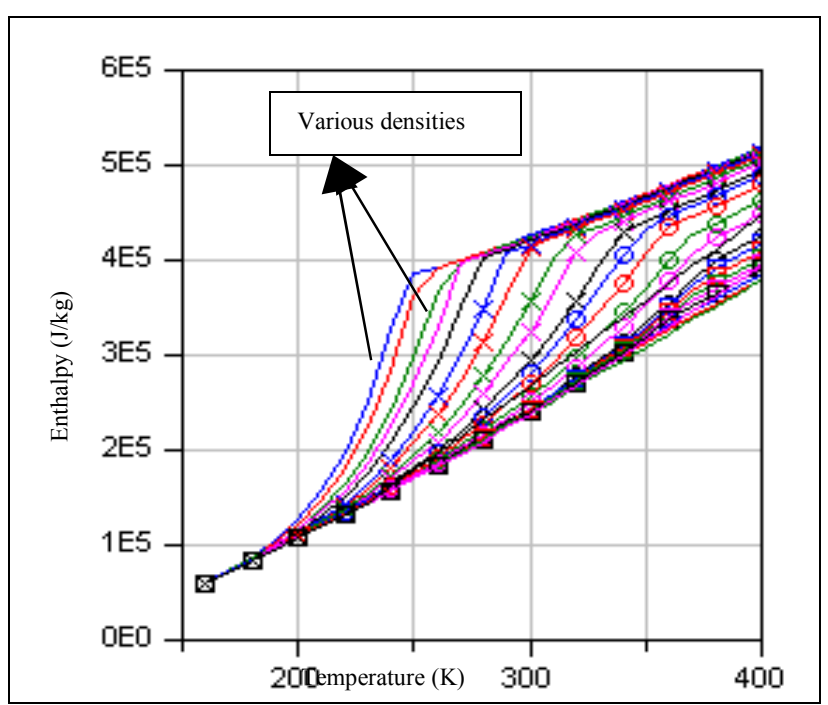

Figure 5 R134a Enthalpy Vs Temperature

\subsubsection{Blower}

The blower delivers the air required by the cabin. Blower mass flow rate is computed by a 2-D look-up table based on fan setting and outlet door position. Both of these inputs are set by the climate system controller. The blower blows the air over the evaporator. The data driven model for the blower is chosen keeping the real time requirements in mind.

\subsubsection{Inlet door}

The inlet door can re-circulate air from the cabin or take fresh air intake. The inlet door model takes in a Boolean signal which indicates the recirculation setting ON or OFF. Based on the re-circulation command from the climate system controller, this door model allows the fresh air to enter into the cabin or re-circulates the cabin air back to the cabin through the blower.

\subsubsection{Heater}

The heater core element of the HVAC system takes heat from the engine coolant and heats the air going into the cabin. The amount of heating can be regulated by adjusting the mixer door that controls the amount of air flow that gets exposed to the heater. The exposed and unexposed air mixes in the region next to the heater. The heating effect of the heater core is implemented using an efficiency term. This heat flow efficiency is given by a look-up table based on the mixer door open ratio. The heater core will be at a temperature dictated by the coolant temperature. The rate at which the air flow gets heated by the heater is controlled by a time constant and the heater efficiency term. 


\subsection{Testing of Models}

The vehicle subsystems and the climate control system models are integrated in the Dymola environment and fundamental validation tests checks were performed. The vehicle was run at a constant speed and the HVAC compressor was switched on and off. The effect of this switching was observed in the cabin temperature. In another test, the time required by the cabin to reach a target temperature at a particular ambient condition with the vehicle being stationary was determined. These tests helped to ascertain the fidelity of the models developed and the corresponding parameterization made.

\section{Real-time Adaptations}

The HVAC components models can become very complex. The calculation of the refrigerant properties in the HVAC component models is itself a computationally expensive calculation. The equations used in the reference [6] are highly non-linear. When the property calculations are written as non-linear functions, differentiating these functions becomes a significant issue. To overcome this problem, tables were made that take density and temperature of the refrigerant as inputs and output the enthalpy, pressure and vapor quality. The condenser and evaporator models can be constructed using an array of refrigerant volumes and refrigerant flow resistance models. Such models were found to be computationally very complex. To avoid this complexity and the associated computational effort, the condenser and evaporator were modeled as single volumes after it was found that the results that were produced with the vehicle model were satisfactory. It was ensured that all the vehicle subsystem models were of the appropriate level of detail to characterize the actual vehicle.

\section{Climate System Controller Model}

The climate system controller model was available as a Simulink S-function. The controller exercises its control action over various components of the HVAC system to maintain the cabin at a set temperature. As the controller is of production standard, only the object code of the source was available. A successful integrated simulation of the developed plant models with the controller itself will mark a milestone. There were no details available about the precise control logic used in the controller. The integration is done based on the available information like input/output signals list of the controller. In many cases, the plant model developer may not get access to the source code of the controller. This method of using the object code for the controller will be very useful in such cases. Except for a few definition files (headers), the object code (binary) was sufficient to download the controller model along with the plant models into the target processor/ machine.

\section{Real-time Simulation}

The first level target of achieving a closed loop simulation of the developed models is accomplished by performing a dynamic simulation of plant and the controller in Simulink. The next level will make sure that the plant models developed in Modelica can be directly used in any Hardware-in-the-Loop testing. There are many practical scenarios where the climate system controller hardware needs to be tested in a real vehicle environment which might not always be possible. This validation testing will trigger the need for HIL simulation where the developed climate system controller hardware will run with the vehicle and climate system components as models in a real-time environment.

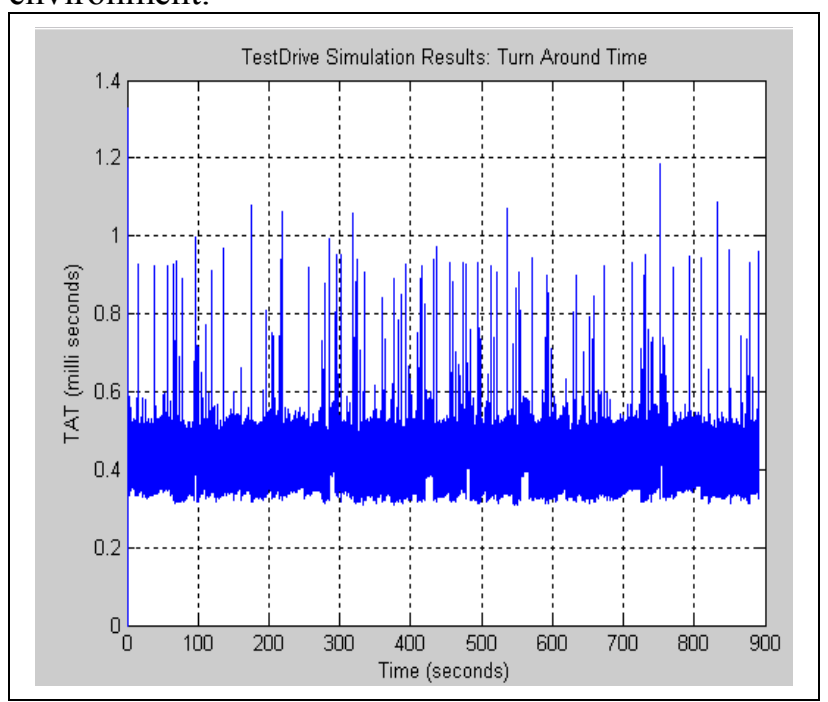

Figure 6 Turn around time of the model

The simulations were carried out for Japanese 1015 mode drive cycle data. The most important metric to be measured for judging the real time capability of models is turn around time. The turn around time for this simulation is well below the chosen sample time of $10 \mathrm{~ms}$ as seen in Figure 6. The plant model results are shown for two different ambient conditions of $40^{\circ} \mathrm{C}$ and $-10^{\circ} \mathrm{C}$. In both the conditions, the controller is able to bring the cabin to the set temperature of $25^{\circ} \mathrm{C}$. In the $40^{\circ} \mathrm{C}$ case, the HVAC compressor is 
switched on and off to control the temperature to $25^{\circ} \mathrm{C}$ (See Figure 7).

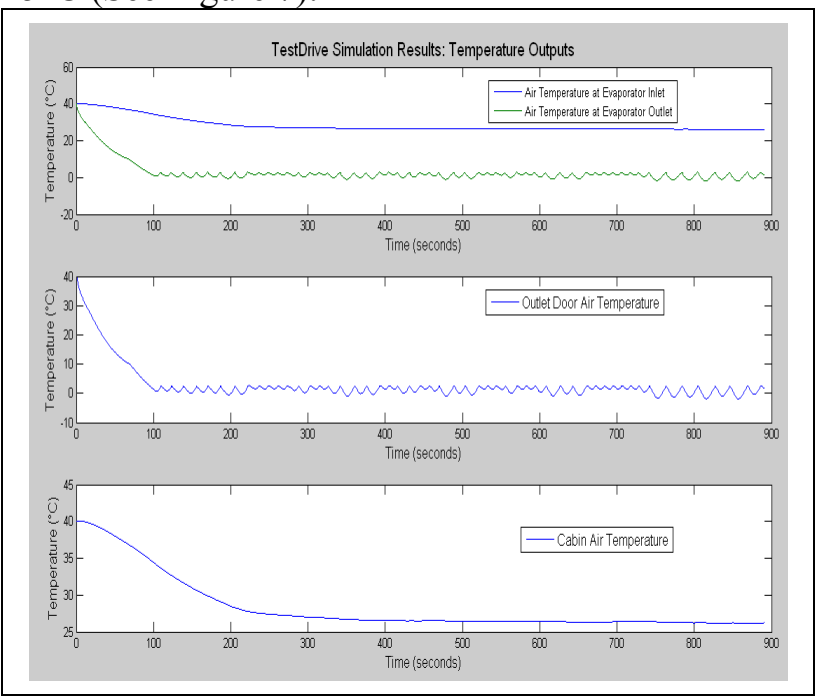

Figure 7 Results for $40^{\circ} \mathrm{C}$ ambient case

In the $-10^{\circ} \mathrm{C}$ case, the heater comes in to play and heats the ambient air to the required $25^{\circ} \mathrm{C}$. In this case, the HVAC compressor was hardly switched on by the controller (see Figure 8).

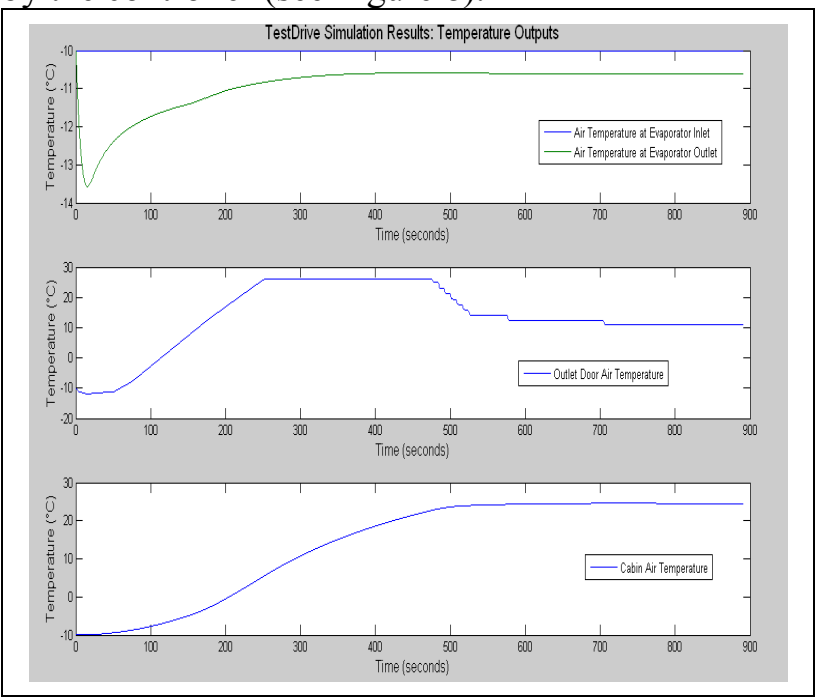

Figure 8 Results for $-10^{\circ} \mathrm{C}$ ambient case

\section{Conclusions}

The exercise of running the closed loop model establishes the compatibility between the developed Modelica plant models with the chosen controller. The closed loop simulation is very beneficial for the following reasons:

- Validation of control strategies and functionality of control elements like heater, inlet door, etc.

- Comparison of different control strategies

- Fuel economy prediction
The real-time simulation of this integrated model in the TestDrive platform confirms that the developed models are amenable to Hardware-in-theLoop simulations. It can be said that the level of fidelity that is captured in these plant models is sufficient for testing climate control logic, fuel economy studies and alternate vehicle configuration sensitivity. These models in the TestDrive hardware can be tested with any climate system controller with suitable input/output connections. In terms of plant modeling, the scope can be increased to add an e-HVAC powered by a battery instead of an engine. The impact of e-HVAC on conventional and hybrid vehicles can also be studied.

\section{Acknowledgment}

This work in parts was supported by Mr. Yutaka Kato of NEAT Co. Ltd., Japan and Dr. Yasunori Yokojima. Authors wish to thank Dr. Michael Tiller for his guidance and support throughout this work.

\section{References}

[1] Öner ARICI, Song-Lin YANG, Daniel HUANG and Emin ÖKER. Computer Model for Automobile Climate Control System Simulation and Application. Int.J. Applied Thermodynamics, Vol.2, (No.2), pp. 59-68, June-1999.

[2] Torge Pfafferott and Gerhard Schmitz. Implementation of a Modelica Library for Simulation of Refrigeration Systems. pp. 197206. Proceedings of the 3rd International Modelica Conference, Linköping, November 3-4, 2003.

[3] Kim, Y. 1993. Two-Phase Flow of HCFC-22 and HFC-134a Through Short-tube Orifices. Ph.D. dissertation, Texas A\&M University.

[4] Hilding Elmqvist, Sven Erik Mattsson, Hans Olsson, Johan Andreasson, Martin Otter, Christian Schweiger and Dag Brück. Realtime Simulation of Detailed Automotive Models. pp. 29-38. Proceedings of the 3rd International Modelica Conference, Linköping, November 3-4, 2003.

[5] J. Bäckman and M. Edvall. Using Modelica and Control Systems for Real-time Simulations in the Pulp. pp. 579-583. Proceedings of the 4th International Modelica Conference, Hamburg, March 7-8, 2005. 
[6] Tillner-Roth, R., Baehr, H.D. An international standard equation of state for the thermodynamic properties of 1,1,1,2-tetrafluoroethane (HFC-134a) for temperatures from $170 \mathrm{~K}$ to $455 \mathrm{~K}$ at pressures up to $70 \mathrm{MPa}$. J. Phys. Chem. Ref. Data 26 (1994) 657-729.

[7] Corberan, J.M. and Melon, M.G. 1998. Modeling of plate finned tube evaporators and condensers working with R134a, Int. J. Refrigeration, Vol. 21, No. 4, pp. 273-284.

[8] M. Tiller, "Introduction to Physical Modeling with Modelica", Kluwer Academic Publishers, ISBN 0-7923-7367-7

[9] Dymola. Dynamic Modeling Laboratory, Dynasim AB, Lund, Sweden, http://www.Dynasim.se

[10] Modelica, http://www.Modelica.org.

[11] OPAL-RT, http://www.opal-rt.com. 